OPEN ACCESS

$$
\begin{array}{r}
\text { Edited by: } \\
\text { Tie-Jun Li, } \\
\text { Second Military Medical } \\
\text { University, China } \\
\text { Reviewed by: } \\
\text { Zhi-Hong Jiang, } \\
\text { Macau University of Science and } \\
\text { Technology, Macau } \\
\text { Tai-jun Hang, } \\
\text { China Pharmaceutical } \\
\text { University, China } \\
\text { Xinhong Wang, } \\
\text { Shanghai University of Traditional } \\
\text { Chinese Medicine, China } \\
\text { *Correspondence: } \\
\text { Yuefen Lou } \\
\text { louyuefen@sina.cn } \\
\text { Guorong Fan } \\
\text { fanguorong@sjtu.edu.cn } \\
\text { These authors have contributed } \\
\text { equally to this work } \\
\text { Specialty section: } \\
\text { Received: } 30 \text { January } 2019 \\
\text { Accepted: 02 May } 2019 \\
\text { Published: } 29 \text { May } 2019 \\
\text { This article was submitted to } \\
\text { Ethnopharmacology, }
\end{array}
$$

Citation:

Meng $X$, Jiang J, Pan $H$, Wu S,

Wang S, Lou Y and Fan G (2019)

Preclinical Absorption, Distribution,

Metabolism, and Excretion of

Sodium Danshensu, One of the

Main Water-Soluble Ingredients in

Salvia miltiorrhiza, in Rats.

Front. Pharmacol. 10:554.

doi: 10.3389/fphar.2019.00554

\section{Preclinical Absorption, Distribution, Metabolism, and Excretion of Sodium Danshensu, One of the Main Water-Soluble Ingredients in Salvia miltiorrhiza, in Rats}

\author{
Xiangguo Meng ${ }^{1 \dagger}$, Jingjing Jiang ${ }^{2 t}$, Hui $\mathrm{Pan}^{3}$, Shengyuan $\mathrm{Wu}^{4}$, Shuowen Wang ${ }^{5}$, \\ Yuefen Lou ${ }^{2 \star}$ and Guorong Fan ${ }^{3,4,5 *}$
}

\begin{abstract}
'Department of Pharmacy, Shanghai University of Medicine and Health Sciences, Shanghai, China, ${ }^{2}$ Department of Pharmacy, Shanghai Fourth People's Hospital, Shanghai, China, ${ }^{3}$ Department of Clinical Pharmacy, Shanghai General Hospital, School of Medicine, Shanghai Jiao Tong University, Shanghai, China, ${ }^{4}$ Laboratory of Drug Metabolism and Pharmacokinetics, School of Medicine, Tongji University, Shanghai, China, ${ }^{5}$ Shanghai Key Laboratory for Pharmaceutical Metabolite Research, School of Pharmacy, Second Military Medical University, Shanghai, China
\end{abstract}

In this study, the absorption, distribution, metabolism and excretion (ADME) of sodium danshensu (Sodium DL- $\beta$-(3, 4-dihydroxyphenyl)lactate), one of the main water-soluble active constituents in Salvia miltiorrhiza, were evaluated in rats. Pharmacokinetic study was evaluated in doses of 15, 30, and $60 \mathrm{mg} / \mathrm{kg}$ after intravenous administration of sodium danshensu. Bioavailability study was evaluated by comparing between $30 \mathrm{mg} / \mathrm{kg}$ (I.V.) and $180 \mathrm{mg} / \mathrm{kg}$ (P.O.) of sodium danshensu. Tissue distribution, metabolism, and excretion were evaluated at $30 \mathrm{mg} / \mathrm{kg}$ (I.V.) of sodium danshensu. Following intravenous administration, sodium danshensu exhibited linear pharmacokinetics in the dose range of $15-60 \mathrm{mg} / \mathrm{kg}$. Sodium danshensu appeared to be poorly absorbed after oral administration, with an absolute bioavailability of $13.72 \%$. The primary distribution tissue was kidney, but it was also distributed to lung, stomach, muscle, uterus, heart, etc. Within $96 \mathrm{~h}$ after intravenous administration, 46.99\% was excreted via urine and $1.16 \%$ was excreted via feces as the parent drug. Biliary excretion of sodium danshensu was about $0.83 \%$ for $24 \mathrm{~h}$. Metabolites in urine were identified as methylation, sulfation, both methylation and sulfation, and acetylation of danshensu. Sodium danshensu can be developed as an injection because of its poor oral bioavailability. In conclusion, sodium danshensu is widely distributed, mainly phase II metabolized and excreted primarily in urine as an unchanged drug in rats.

Keywords: sodium danshensu, pharmacokinetics, LC-MS/MS, ADME, bioavailability study

\section{BACKGROUND}

Salvia miltiorrhiza, named Danshen in Chinese, is one of the most versatile traditional Chinese medicinal herbs. It has been widely used to treat and prevent cardio vascular disease, hyperlipidemia and cerebro vascular disease throughout the world (Cheng 2007; Wang et al., 2017). Up to today, more than 70 compounds have been isolated and a wide spectrum of secondary metabolites 
has been identified from Salvia miltiorrhiza, including diterpenoid quinines, hydrophilic phenolic acids and essential oil constituents (Li et al., 2009). It is generally known that lipophilic diterpenoid quinines and hydrophilic phenolic acids are the main bioactive components in Salvia miltiorrhiza (Su et al., 2015).

Danshensu, also named as salvinanic acid, is the main water-soluble phenolic acids in Salvia miltiorrhiza. It has been reported that danshensu exhibited several pharmacological activities such as cardioprotective effect by inhibiting L-type calcium channels (Song et al., 2016), radioprotective effect by scavenging reactive oxygen species (Guo et al., 2013), protection of vascular endothelial cells by an antioxidative mechanism (Zhao et al., 2012), protection on liver injured by an antioxidative mechanism (Wang et al., 2007), etc.

Because danshensu is unstable in the nature, it was transformed into its sodium salt, sodium danshensu (sodium DL- $\beta-(3,4-$ dihydroxyphenyl)lactate, Figure 1). Sodium danshensu has the same effectiveness as danshensu (Wang and $\mathrm{Xu}, 2005$; Wang et al., 2007; Zhao et al., 2013; Jia et al., 2018). Due to its potential pharmacological activities, sodium danshensu has attracted more and more attention recently and is being investigated as a new drug (Zhou et al., 2009; Wei et al., 2018). In order to investigate the pharmacokinetics of sodium danshensu, several biological analytical methods have been reported ( $\mathrm{Li}$ et al., 2008; Zhan et al., 2008; Liu et al., 2010, 2014; Fu et al., 2014; Zheng et al., 2015), and a high-throughput, simple, sensitive, selective and reliable LC-MS/MS method was developed and validated in our laboratory before (Jiang et al., 2017). Although many researchers published several articles about pharmacokinetic study of sodium danshensu in rats, detailed pharmacokinetic characteristics including absorption, distribution, metabolism, and elimination (ADME) of sodium danshensu have not been reported. In this study, we extensively investigated the preclinical ADME and bioavailability study of sodium danshensu in rats.

\section{MATERIALS AND METHODS}

\section{Chemicals and Reagents}

Sodium danshensu (purity 98.5\%) was made and provided by the Prof. Chuan Zhang of School of Pharmacy, Second Military Medical University, Shanghai (Zhang et al., 2010). Ketoprofen (purity $\geq 95 \%$ ), the internal standards (IS), was purchased from the National Institute for Food and Drug Control (Beijing, China). HPLC-grade methanol and acetonitrile were purchased from Merck (Darmstadt, Germany). Ethyl acetate was obtained

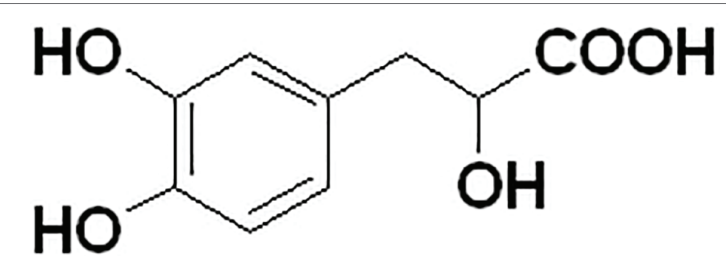

FIGURE 1 | Chemical structure of (D/L) sodium danshensu. from Shanghai Sheng De Chemical Co., Ltd. (Shanghai, China). Formic acid was HPLC grade and purchased from TEDIA Company (Fairfield, CA, USA). Deionized water was prepared by using the Milli-Q Plus Ultrapure Water System (Millipore Corporation, Bedford, MA). All other chemicals used in this study were of the highest quality available.

\section{Animals}

Sprague-Dawley (SD) rats (male and female equally, 180-220 kg, 6-8 weeks) were purchased from the Shanghai Slack Experimental Animal co., LTD (Shanghai, China) and were acclimated in laboratory for at least 1 week prior to the study. The animal room was controlled to maintain a temperature of $18-25^{\circ} \mathrm{C}$ and relative humidity of $20-60 \%$. The animals were housed under a 12-h light/dark cycles and allowed free access to food and water.

\section{Pharmacokinetic Study}

Rats were fasted overnight before dosing and $6 \mathrm{~h}$ after dosing. For the pharmacokinetic study and bioavailability study, 24 rats were randomly divided into four groups $(n=24,6$ per group) and were respectively designed as different dosages (15, 30, $60 \mathrm{mg} / \mathrm{kg}$ I.V., $180 \mathrm{mg} / \mathrm{kg}$ P.O.).

Blood samples were serially collected from orbital venous plexus into heparinized tubes pre-dose and at $0,0.08,0.17$, $0.33,0.67,1.0,1.5,2.0,3.0,4.0,6.0,8.0$, and $12.0 \mathrm{~h}$ after dosing for the three intravenous injection groups. For the oral administration group, blood samples were serially collected from orbital venous plexus into heparinized tubes pre-dose and at $0.08,0.17,0.25,0.5,0.75,1.0,1.5,2.0,3.0,4.0,6.0$, 8.0, and $12.0 \mathrm{~h}$ after dosing. All rats were transfused normal saline after drawing blood. Plasma was then separated from blood by centrifuging at 3,500 rpm for $10 \mathrm{~min}$ immediately and transferred to another tubes and stored at $-80^{\circ} \mathrm{C}$ before analysis.

\section{Distribution}

In tissue distribution study, rats ( $n=36,6$ per group) were randomly divided into six groups and sex distribution equally per group, intravenously injected with a single dose of $30 \mathrm{mg} / \mathrm{kg}$ sodium danshensu. At $0.17,0.67,1,2,4$, and $6 \mathrm{~h}$ after dosing, rats were anesthetized with diethyl ether and sacrificed. Tissues (heart, liver, spleen, lung, kidney, brain, stomach, intestinal, fat, muscle, testis, ovary, and uterus) were excised immediately, rinsed with saline, dried with filter paper, weighed, and homogenized in saline. Tissue homogenates were stored at $-80^{\circ} \mathrm{C}$ before analysis.

\section{Metabolism}

Metabolites were analyzed in rat urine with intravenously injected with a single dose of $30 \mathrm{mg} / \mathrm{kg}$ sodium danshensu by LC-MS/MS. An aliquot of $500 \mu \mathrm{l}$ of urine was acidified with $250 \mu \mathrm{l}(3 \mathrm{~mol} / \mathrm{L})$ of $\mathrm{HCl}$ and vortexed. The mixture was then loaded to activate C18 SPE cartridges, washed with $1 \mathrm{ml}$ of water, and eluted with $1 \mathrm{ml}$ of $80 \%$ methanol. The eluant was then evaporated to dryness under a nitrogen gas stream 
and reconstituted with $100 \mu \mathrm{l}$ of mobile phase, and centrifuged at $12,000 \mathrm{rpm}$ for $10 \mathrm{~min}$. The supernatant was then transferred and applied for LC-MS/MS detection.

\section{Excretion}

Urine and feces samples were obtained from rats $(n=6)$ intravenously administrated with intravenously injected with a single dose of $30 \mathrm{mg} / \mathrm{kg}$ sodium danshensu and housed individually in metabolic cages equipped with a urine and feces separator. The urine and fecal samples were collected pre-dose and at $0-2,2-4,4-6,6-8,8-12,12-24,24-36,36-48$, $48-60,60-72,72-84$, and $84-96 \mathrm{~h}$ after doing. Urine and feces were stored at $-80^{\circ} \mathrm{C}$ before analysis.

In biliary excretion study, SD rats $(n=6)$ were anesthetized with urethane $(1.2 \mathrm{~g} / \mathrm{kg}$, ip) and the bile duct was cannulated. After intravenous administration with a single dose of $30 \mathrm{mg} / \mathrm{kg}$ sodium danshensu, bile was collected at $2 \mathrm{~h}$ intervals for the first $12 \mathrm{~h}$ and at $24 \mathrm{~h}$. Bile samples were stored at $-80^{\circ} \mathrm{C}$ before analysis.

\section{Sample Preparation}

In the present study, sodium danshensu and IS were extracted from rat plasma by using a liquid-liquid extraction method. A $25 \mu \mathrm{l}$ aliquot of IS solution containing $40.16 \mathrm{ng} / \mathrm{ml}$ of ketoprofen was added to a $50 \mu \mathrm{l}$ aliquot of rat plasma and then vortexed and acidified with $20 \mu \mathrm{l}$ of $3 \mathrm{~mol} / \mathrm{L} \mathrm{HCl}$. The mixture was then extracted with $1 \mathrm{ml}$ of ethyl acetate, vortexed, and centrifuged at 5,000 rpm for $10 \mathrm{~min}$. A $800 \mu \mathrm{l}$ aliquot of the supernatant was then transferred to another tube and evaporated to dry. The residue was then reconstituted with $50 \mu \mathrm{l}$ of mobile phase and centrifuged at $12,000 \mathrm{rpm}$ for $10 \mathrm{~min}$. The supernatant was then transferred to LC-MS/ MS detection.

In the tissue distribution study, $50 \mu \mathrm{l}$ IS solution $(52.6 \mathrm{ng} / \mathrm{ml})$ was added to $100 \mu \mathrm{l}$ tissue homogenates respectively. The mixed samples were vibrated $30 \mathrm{~s}$ and acidified with $50 \mu \mathrm{l}$ of $3 \mathrm{~mol} / \mathrm{L}$ $\mathrm{HCl}$. The rest of the experimental operation was same as the plasma samples' process.

In the excretion study, feces were prepared by ultrasonic extraction in $0.9 \% \mathrm{NS}$ for $5 \mathrm{~min}$ and vortex for $3 \mathrm{~min}$. After centrifuging at 3,500 rpm for $10 \mathrm{~min}, 100 \mu \mathrm{l}$ supernatant fecal extract was prepared immediately before analysis. The sample preparation for urine, bile and fecal extract were the same to plasma samples. Except that the concentrations of IS or the volumes of $\mathrm{HCl}$ were different. A $50 \mu \mathrm{l}$ aliquot of IS solutions at concentrations of $312,103.2$, and $103.2 \mathrm{ng} / \mathrm{ml}$ were added to a $100 \mu \mathrm{l}$ aliquot of urine, bile, or feces samples, respectively. And all were acidified with $50 \mu \mathrm{l}$ of $3 \mathrm{~mol} / \mathrm{L} \mathrm{HCl}$ by $30 \mathrm{~s}$.

\section{LC-MS/MS Conditions}

The method combining liquid chromatography with electrospray ionization tandem mass spectrometry to determine the sodium danshensu in plasma was developed and validated as described previously (Yu et al., 2011). Analysis was performed using a VARIAN 1200L HPLC-MS system equipped with VARIAN ProStar 210 pump, VARIAN ProStar 410 autosampler, VARIAN
1200L Quadrupole MS/MS, and VARIAN MS 6.8 workstation. The analytes were separated by a Diamonsil $\mathrm{C}_{18}$ column $(200 \mathrm{~mm} \times 4.6 \mathrm{~mm}, 5 \mu \mathrm{m})$ at $25^{\circ} \mathrm{C}$ with a mobile phase consisting of methanol and $0.1 \%$ formic acid in water (80:20, $\mathrm{v} / \mathrm{v}$ ) at a flow rate of $0.8 \mathrm{ml} / \mathrm{min}$, and $2 / 5$ of flow was entered into mass spectrometer. Injection volume was set at $20 \mu \mathrm{l}$ and analysis time was set at $9 \mathrm{~min}$. Electrospray ionization was operated in a negative ion mode. The optimized precursor-toproduct ion transitions were monitored at $\mathrm{m} / \mathrm{z} \quad 197-135$ and 253-209 for sodium danshensu and IS, respectively. Sourcedependent parameters were set as follows: needle $-4,700 \mathrm{~V}$; shield $-150 \mathrm{~V}$; nebulizing gas flow $3.31 \mathrm{Mpa}$; drying gas $250^{\circ} \mathrm{C}$, $1.38 \mathrm{MPa}$; capillary voltage $-40 \mathrm{~V}$; and collision energy 18.0 and $5.5 \mathrm{~V}$ for sodium danshensu and IS.

\section{Data Analysis}

Pharmacokinetic parameters of sodium danshensu were analyzed by using the BAPP software (version 3.1, Center of Drug Metabolism and Pharmacokinetics, China Pharmaceutical University, Nanjing, China) with a noncompartmental model. All data are presented as mean \pm SD. The absolute bioavailability $(\% F)$ of sodium danshensu was estimated via the ratio of $\mathrm{AUC}_{0-\infty}$ after oral and intravenous administration.

\section{RESULTS}

\section{Pharmacokinetic Results}

The pharmacokinetic profiles of sodium danshensu in rats following intravenous administration of sodium danshensu at doses of 15, 30, and $60 \mathrm{mg} / \mathrm{kg}$ are shown in Figure 2, and relevant pharmacokinetic parameters obtained are listed in Table 1. Plasma concentrations of sodium danshensu decreased quickly with elimination half time $\left(t_{1 / 2}\right)$ of $2.76 \pm 0.72$, $3.00 \pm 0.31$, and $2.64 \pm 0.44 \mathrm{~h}$, respectively. The $\mathrm{AUC}_{0-12}$ values were determined to be $12.67 \pm 1.40,34.27 \pm 2.49$, and

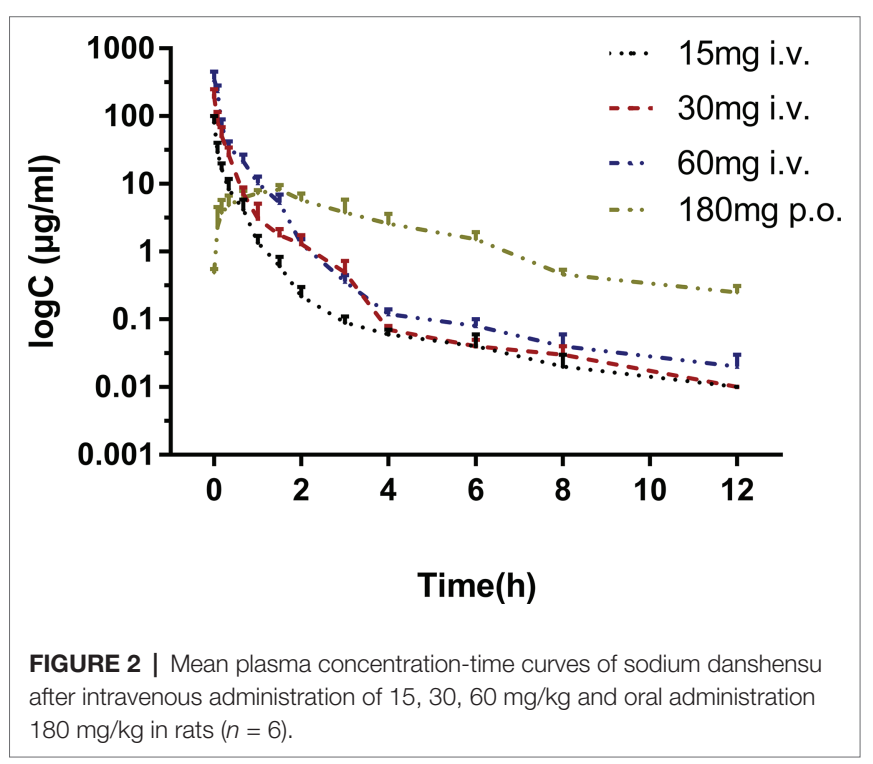


TABLE 1 | Pharmacokinetic parameters of sodium danshensu in rats after intravenous administration of 15, 30, and $60 \mathrm{mg} / \mathrm{kg}$ and oral of $180 \mathrm{mg} / \mathrm{kg}(n=6)$.

\begin{tabular}{|c|c|c|c|c|c|}
\hline Parameters & Units & 15 mg/kg (I.V.) & 30 mg/kg (I.V.) & 60 mg/kg (I.V.) & 180 mg/kg (P.O.) \\
\hline$T_{\max }$ & h & - & - & - & $1.40 \pm 0.30$ \\
\hline$C_{\max }$ & $\mu \mathrm{g} / \mathrm{ml}$ & $81.18 \pm 19.26$ & $195.32 \pm 53.15$ & $349.32 \pm 104.85$ & $8.76 \pm 0.85$ \\
\hline$t_{1 / 2}$ & h & $2.76 \pm 0.72$ & $3.00 \pm 0.31$ & $2.64 \pm 0.44$ & $2.35 \pm 0.25$ \\
\hline $\mathrm{AUC}_{0-12}$ & $\mu \mathrm{g} \mathrm{h} / \mathrm{ml}$ & $12.67 \pm 1.40$ & $34.27 \pm 2.49$ & $67.70 \pm 11.71$ & $27.40 \pm 4.54$ \\
\hline $\mathrm{AUC}_{0-\infty}$ & $\mu \mathrm{g} \mathrm{h} / \mathrm{ml}$ & $12.73 \pm 1.40$ & $34.31 \pm 2.49$ & $67.76 \pm 11.72$ & $28.24 \pm 4.57$ \\
\hline MRT & h & $0.46 \pm 0.07$ & $0.41 \pm 0.04$ & $0.39 \pm 0.04$ & $3.35 \pm 0.23$ \\
\hline
\end{tabular}

$67.70 \pm 11.71 \mu \mathrm{g} \mathrm{h} / \mathrm{ml}$. The results of the variance analysis showed that the $t_{1 / 2}$ and MRT had no significant differences among different groups $(p>0.05)$. It seems that exposure increases with dosages in the dose range of $15-60 \mathrm{mg} / \mathrm{kg}$ and the relationship between $\mathrm{AUC}_{0-12}$ and dose was reflected in the liner correlation coefficient $\left(r^{2}=0.9338\right)$.

The absolute bioavailability was calculated by comparing between oral and intravenous administration of sodium danshensu in another study. The mean plasma concentrationtime curves in rats following oral administration are also illustrated in Figure 2, and the pharmacokinetic parameters are listed in Table 1. $T_{\max }$ after oral administration is $1.40 \pm 0.30 \mathrm{~h}$, indicating that the absorption of sodium danshensu from the gastrointestinal tract is in a moderate speed. The absolute oral bioavailability of sodium danshensu in rats was calculated to be $13.72 \%$, indicating poor absorption following oral administration in rats.

\section{Tissue Distribution}

Following intravenous administration, sodium danshensu was distributed throughout the body. The concentration of sodium danshensu was detectable in all tissues studied, including heart, liver, spleen, lung, kidney, brain, stomach, intestinal, fat, muscle, testis, ovary, and uterus, as showed in Figure 3. Peak levels reached at $0.17 \mathrm{~h}$ in all tissues and decreased gradually in most of the tissues at $6 \mathrm{~h}$ pose-dose. The primary distribution site was kidney, followed by uterus, lung, muscles, and stomach. Kidney distribution of sodium danshensu is 9-fold to 388 -fold higher than other tissues at $0.17 \mathrm{~h}$.

\section{Metabolite Profiling in Rat Urine}

Rat urine samples were collected from rats administrated with sodium danshensu and analyzed for metabolite profiling. Total ion chromatogram (TIC) of dosed rat urine was shown in Figure 4. There are seven metabolites speculated in rat urine, named as M1 to M7. Extracted ion chromatograms (EIC) of seven metabolites were displayed in Figures 5-8. M1-M3 were metabolites with molecular ions at $\mathrm{m} / \mathrm{z} 211,14$ amu greater than that of danshensu $(\mathrm{m} / \mathrm{z}$ 197). This additional evidence supported that M1-M3 were methylated metabolites of danshensu. Molecular ion of M4 was at $\mathrm{m} / \mathrm{z} 277,80 \mathrm{amu}$ greater than that of danshensu, indicating that M4 was a sulfated metabolite of danshensu. M5 and M6 were metabolites with $\mathrm{m} / \mathrm{z} 291,94 \mathrm{amu}$ greater than that of danshensu, presumed to be methylated and sulfated danshensu. Molecular ion of M7 was at m/z 239, $42 \mathrm{amu}$ greater than that of danshensu, indicating that M7 was an acetylated metabolite of danshensu. Possible metabolic pathways of sodium danshensu in rats were shown in Figure 9.

\section{Excretion of Sodium Danshensu}

Excretion of sodium danshensu into urine and feces after a single intravenous administration of sodium danshensu $(30 \mathrm{mg} / \mathrm{kg})$ to rats is presented in Figure 10. Urinary recovery of total sodium danshensu for the first $12 \mathrm{~h}$ period is about $46.57 \pm 19.25 \%$ of the administrated dose, and the value for $96 \mathrm{~h}$ period was $46.99 \pm 19.37 \%$. Compared with urinary excretion, feces excretion of sodium danshensu is very small, only about $1.16 \pm 0.26 \%$ of the dose reclaimed in the feces within $96 \mathrm{~h}$ post-dose.

Biliary excretion of sodium danshensu after a single intravenous administration of sodium danshensu $(30 \mathrm{mg} / \mathrm{kg})$ is also shown in Figure 10. Total excretion into bile over $24 \mathrm{~h}$ is about $0.83 \pm 0.11 \%$ of the administrated dose. In general, urine was the predominant route of elimination of the prototype drug, accounting for $46.99 \%$ of the administrated dose. Recovery of sodium danshensu in feces and bile were very small, with about 1.16 and $0.83 \%$ of the dose, respectively.

\section{DISCUSSION}

In this study, the preclinical absorption, distribution, metabolism, and excretion of sodium danshensu in rats was investigated. Sodium danshensu was poorly absorbed following a single oral administration, with an absolute bioavailability of $13.72 \%$. It is generally known that many active compositions of Chinese Herbal Medicine have low bioavailability, which may be caused by reasons such as physical and chemical properties of drugs, damaged by intestinal environment, metabolized by intestinal microflora, metabolized by enzyme expressed in intestinal epithelial cell, efflux transported by transporters expressed in intestinal epithelial cell, and so on. Previous studies indicated that sodium danshensu was a substrate of P-gp (Yu et al., 2011). Besides, poor bioavailability, ranged from 11.09 to $28 \%$, was also reported by other researchers (Zhou et al., 2009; Wang et al., 2015). In follow-up experiments, such as the tissue distribution, metabolites, and excretion study, sodium danshensu was given by intravenous administration.

Tissue distribution study indicated that sodium danshensu was highly distributed in the kidney. Wang reported that 


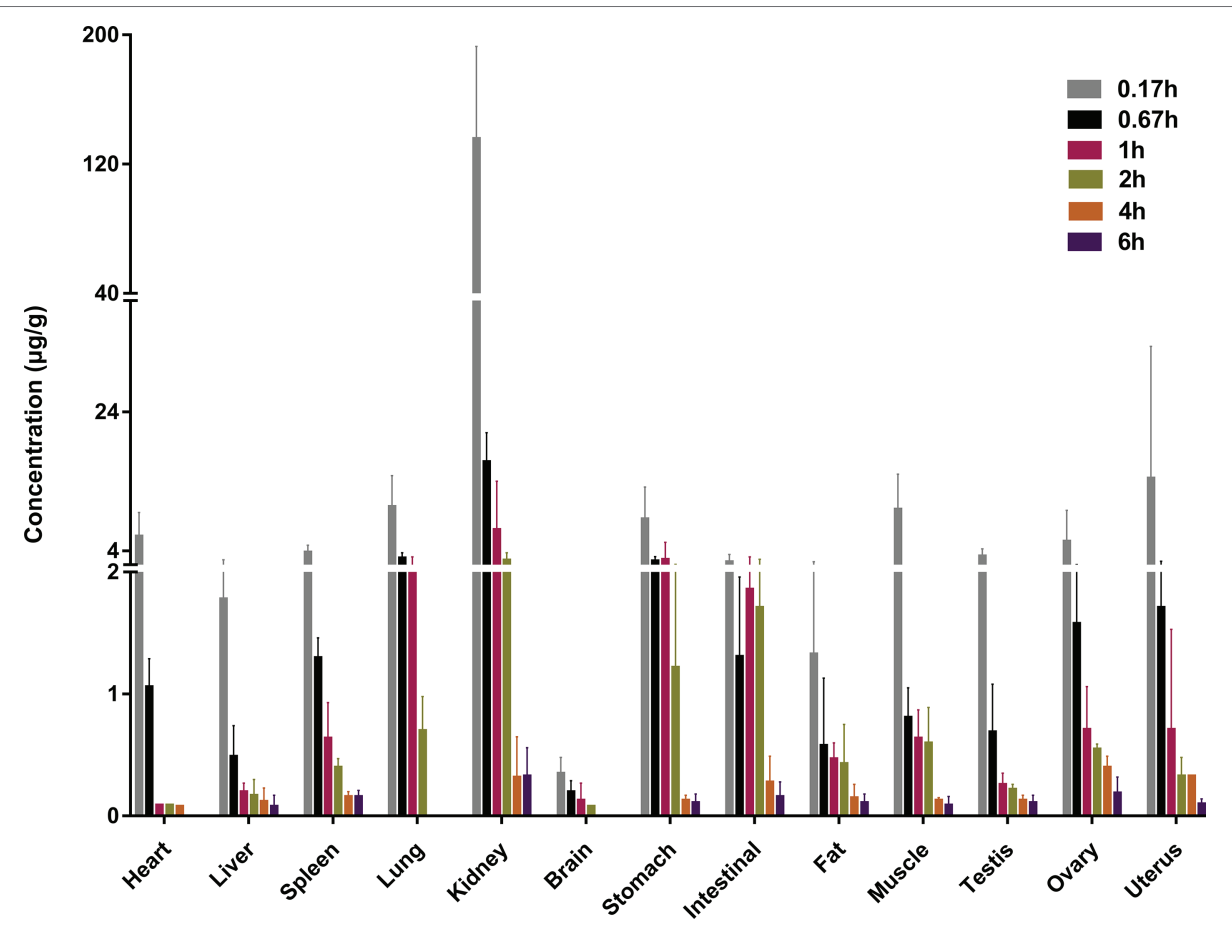

FIGURE 3 | Tissue concentration of sodium danshensu at different time points after intravenous administration of $30 \mathrm{mg} / \mathrm{kg}$ in rats.

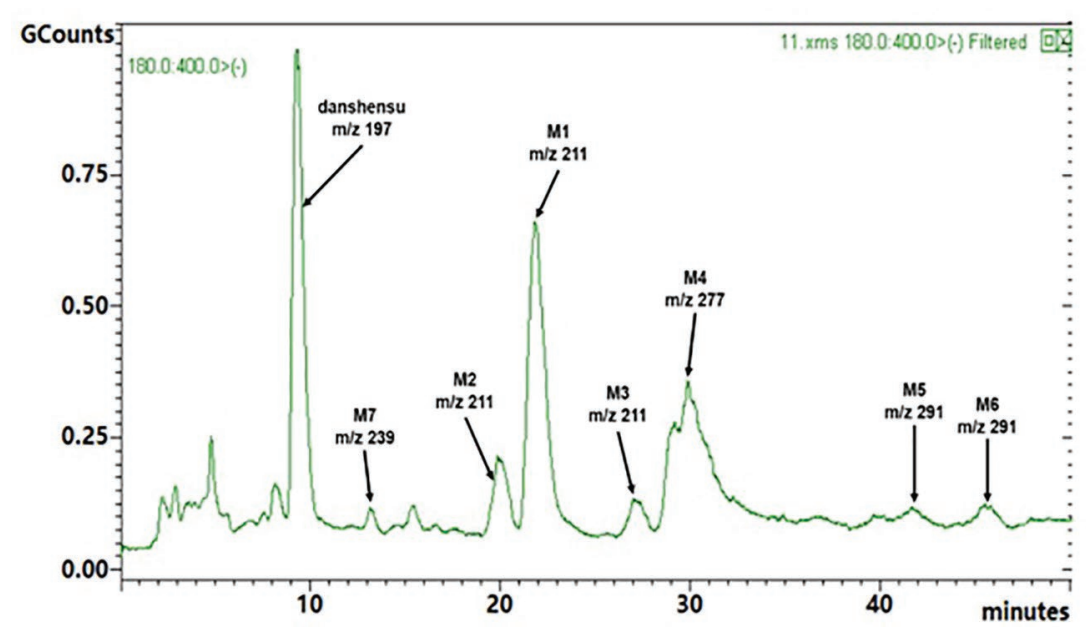

FIGURE 4 | TIC of rat urine sample after intravenous administration of $30 \mathrm{mg} / \mathrm{kg}$ sodium danshensu.

danshensu demonstrated a competitive inhibition towards organic anion transporters 1 and 3 (Wang and Sweet, 2013). However, no researcher has investigated whether danshensu is a substrate of organic anion transporter 1 or 3 . Organic anion transporters 1 and 3 specifically expressed in the kidney mediated the renal accumulation of many drugs. Our study found that danshensu was highly accumulated in rat kidney. In addition, danshensu is an acidic compound, which is in accordance with substrate characteristics of organic anion transporter 1 or 3. It can be speculated that danshensu may be a substrate of organic anion transporter 1 or 3 , which depends further investigation. From the perspective of cardiovascular pharmacological, the tissue distribution study of sodium danshensu also indicated that kidney may be the important target organs exerting pharmacodynamic effects. Preliminary research demonstrated that Danshen has an anti-hypertensive effect through the inhibition of the renin angiotensin system (Kang et al., 2002). According to the theory of traditional Chinese medicine, Danshen could promote blood circulation and dieresis by regulation the AQP2 in kidney (Dong et al., 2014). So, this tissue distribution study could provide reference for further pharmacological research. 


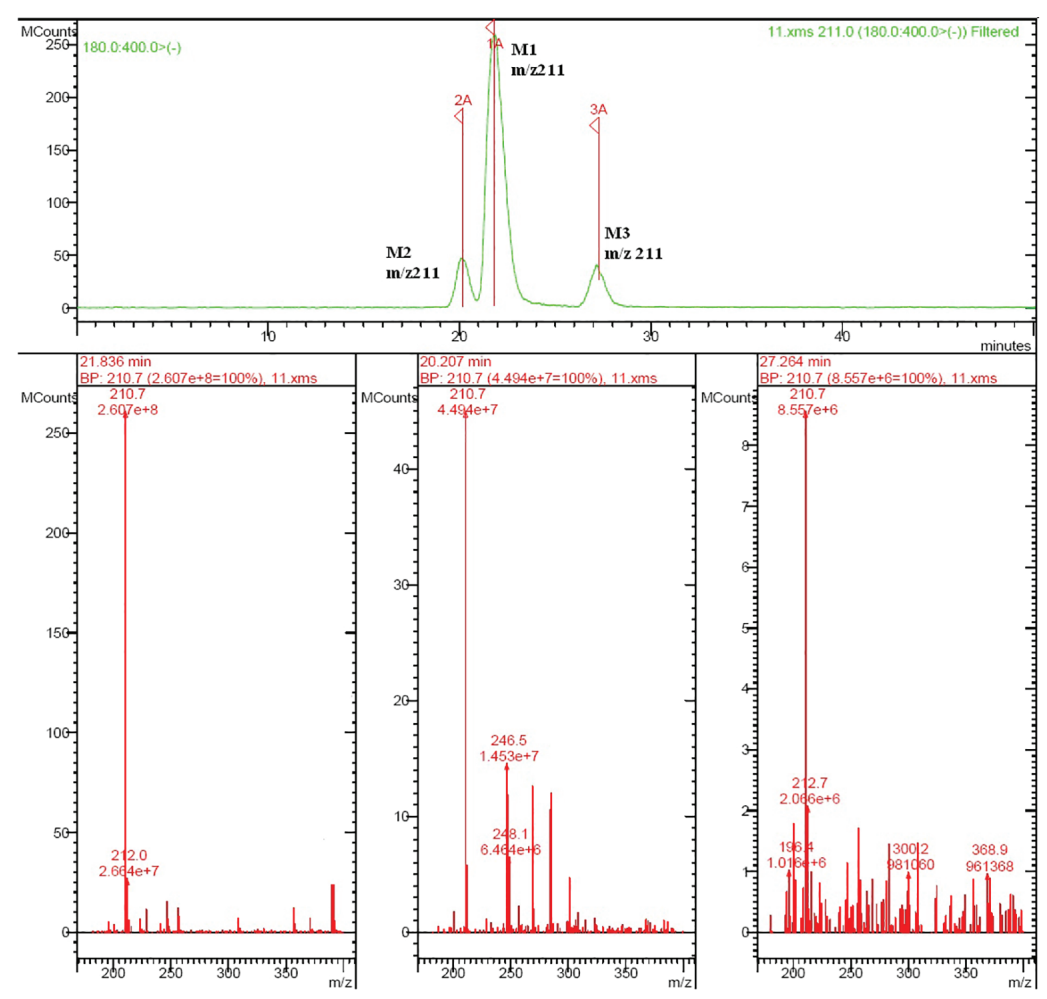

FIGURE 5 | EIC of rat urine sample at m/z 211.

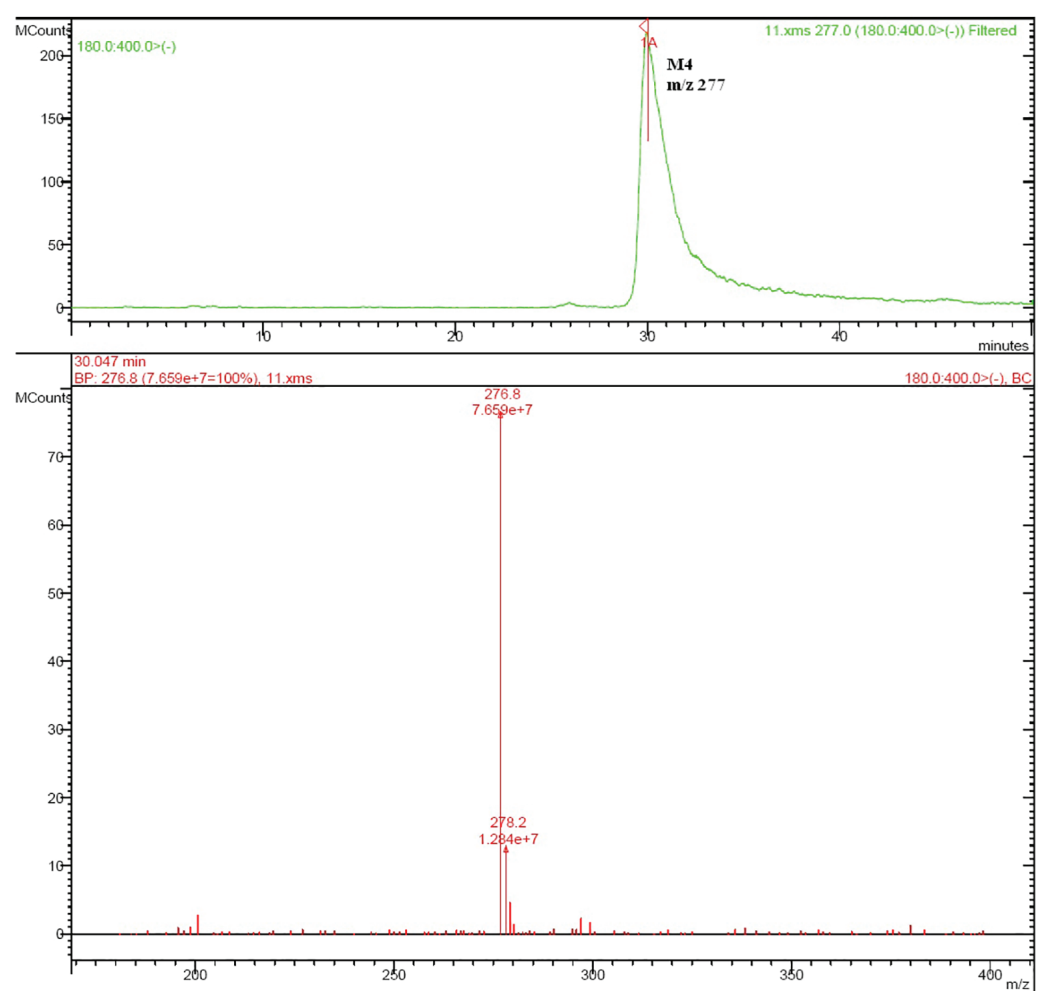

FIGURE 6 | EIC of rat urine sample at m/z 277. 
Mong et al.

Preclinical ADME of Sodium Danshensu in Rats

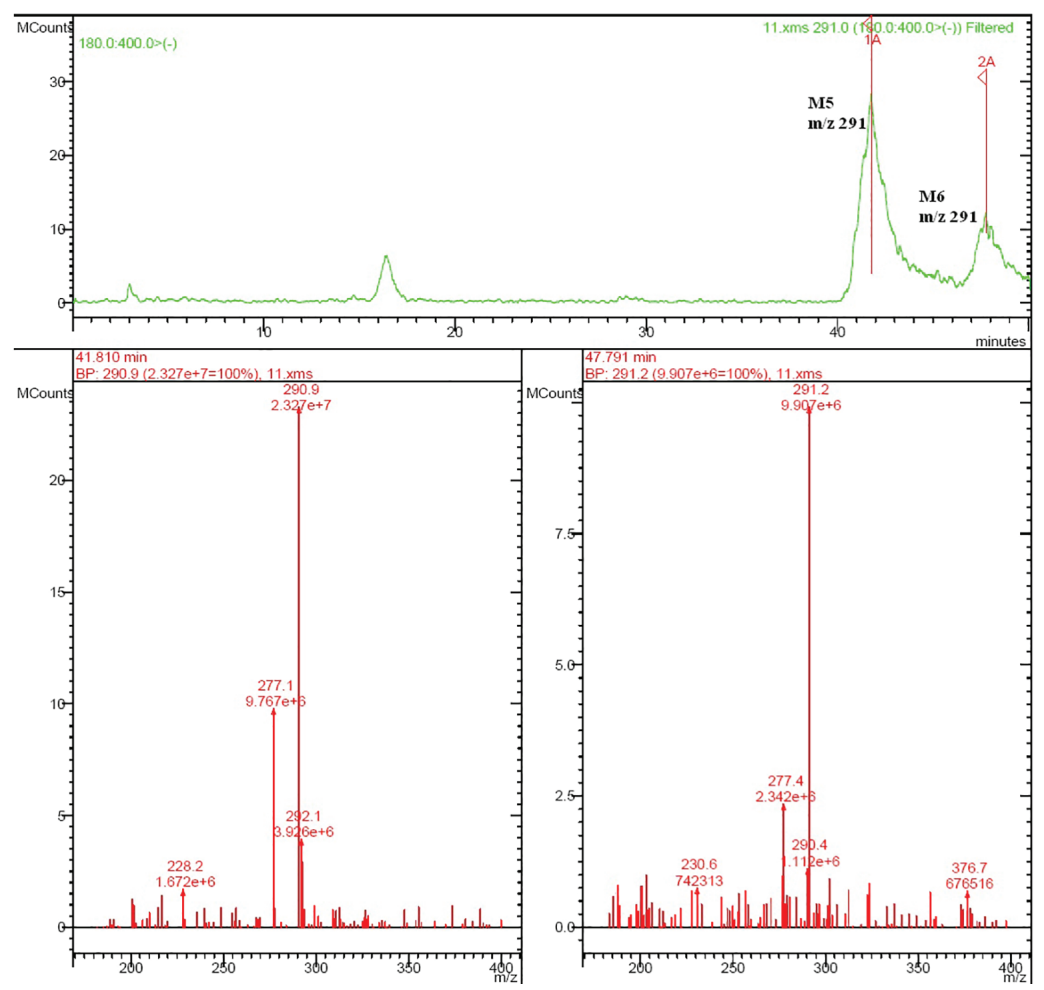

FIGURE 7 | EIC of rat urine sample at m/z 291.

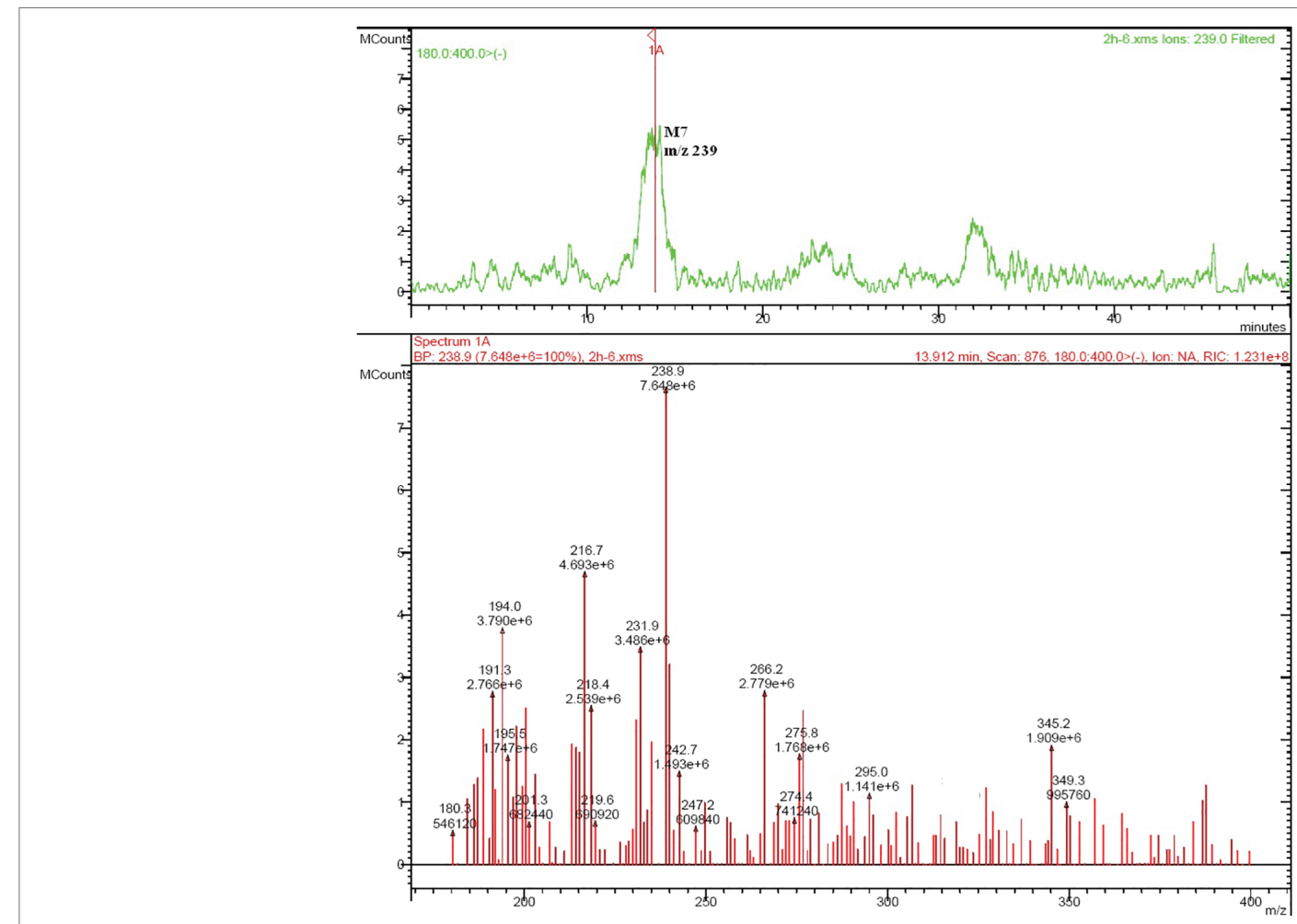

FIGURE 8 | EIC of rat urine sample at m/z 239.

Frontiers in Pharmacology | www.frontiersin.org

7

May 2019 | Volume 10 | Article 554 


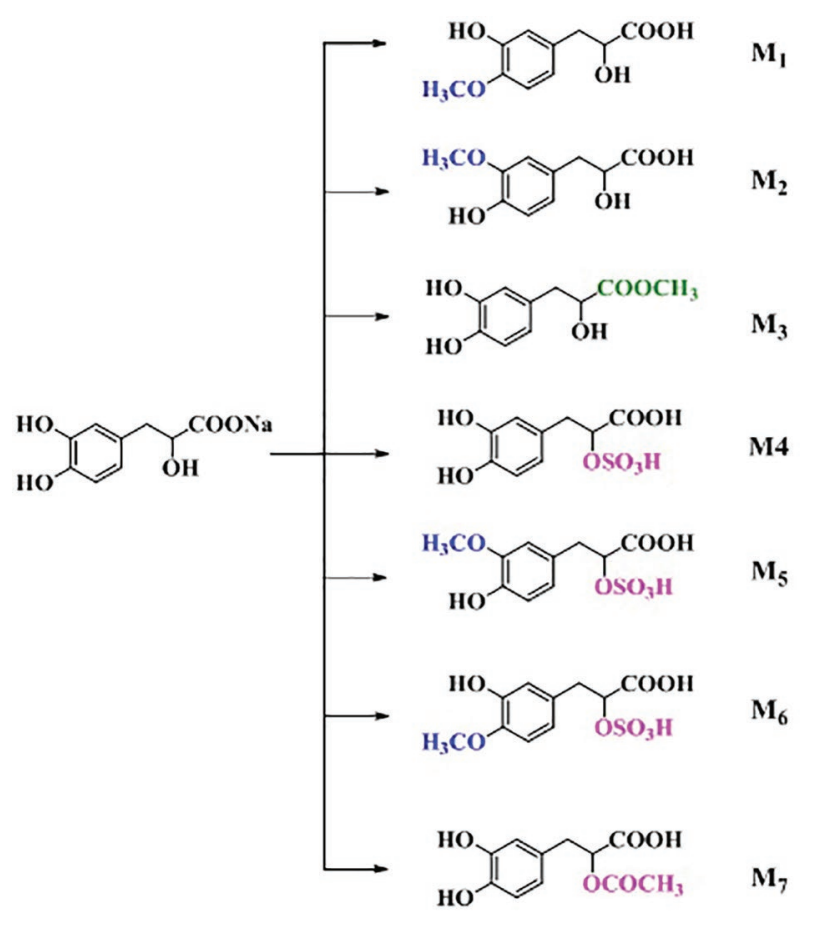

FIGURE 9 | Possible metabolic pathways of sodium danshensu in rats.
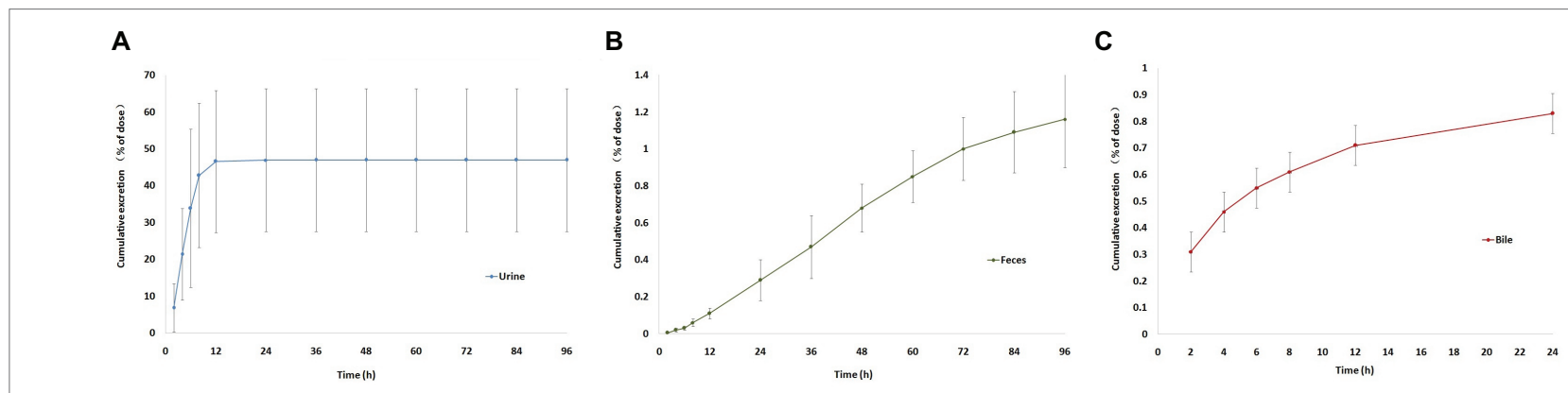

FIGURE 10 | Cumulative excretion of sodium danshensu in rat after intravenous administration of $30 \mathrm{mg} / \mathrm{kg}$. (A) Cumulative excretion of sodium danshensu in rat urine. (B) Cumulative excretion of sodium danshensu in rat feces. (C) Cumulative excretion of sodium danshensu in rat bile.

There are seven sodium danshensu metabolites speculated in rat urine in this study, including three methyl-danshensu metabolites, one danshensu sulfate, one methyl-danshensu sulfate, and one acetyl- danshensu. Due to lack of reference substances, qualitative rather than quantitative analysis was conducted. All metabolites were deduced by their precursor ion and product ions (data not shown). Three hydroxyls exist in the structure of SAA, which may be served as methylation binding sites. $\mathrm{Xu}$ found that methylated metabolites of sodium danshensu displayed even higher antioxidant activity against lipid peroxidation in rat liver in vitro. Methylated metabolites may contribute to the pharmacological activities of SAA (Xu et al., 2014). Shen found five metabolites in rat plasma dosed with danshensu $(20 \mathrm{mg} / \mathrm{kg}$, IV), including danshensu mono-glucuronide, monomethyl-danshensu monoglucuronide, mono-methyl-danshensu, dimethyl-danshensu, and dimethyldanshensu-monoglucuronide (Shen et al., 2009). In another study, Gu JF found five metabolites in rat urine dosed with danshensu, including two danshensu sulfate, one methyldanshensu sulfate, one danshensu mono-glucuronide, and one monomethyl-danshensu mono-glucuronide ( $\mathrm{Gu}$ et al., 2014).

Urinary, fecal, and bile excretion of sodium danshensu in rats after intravenous administration of sodium danshensu have not been reported yet. In this study, the majority of sodium danshensu was found to be excreted in rat urine, with approximately $46.99 \%$ of administrated dose recovered in rat urine in $96 \mathrm{~h}$. Furthermore, urinary excretion of sodium danshensu was concentrated in the first $12 \mathrm{~h}$, with about $46.57 \%$ of sodium danshensu, and indicated that sodium danshensu can be excreted quickly and might not result in accumulation in vivo. 
Excretion of sodium danshensu in rat feces and bile was relatively low, with about 1.16 and $0.83 \%$ of administrated dose. It means that feces and bile play minor role in the elimination of sodium danshensu.

\section{CONCLUSIONS}

In summary, this study provides a comprehensive delineation of sodium danshensu absorption, distribution, metabolism, and elimination profiles in rats. The data demonstrates that sodium danshensu is poorly absorbed, widely distributed, bio-transformed through several metabolic pathways, and excreted mainly in rat urine.

\section{ETHICS STATEMENT}

All pharmacokinetics studies that involved SD rats adhered to the International Guiding Principles for Biomedical Research Involving Animals, as revised by the International Council for

\section{REFERENCES}

Cheng, T. O. (2007). Cardiovascular effects of Danshen. Int. J. Cardiol. 121, 9-22. doi: 10.1016/j.ijcard.2007.01.004

Dong, X. J., Guo, L. F., Yao, R., Xue, S. Y., and Li, F. (2014). Relationship between regulation effect of Salvia miltiorrhiza on AQP2 in kidney and promoting blood circulation and dieresis. Zhongguo Zhong Yao Za Zhi 39, 3162-3165. doi: $10.4268 / \mathrm{cjcmm} 20141631$

Fu, S., Li, L., Qiu, F., Wang, B., Du, W., and Feng, L. (2014). Application of a simple and rapid LC-MS/MS method for determination of danshensu in human plasma for an oral pharmacokinetic study of Danshen granules in chinese healthy subjects. Anal. Methods 6, 1956-1962. doi: 10.1039/C3AY42134A

Gu, J. F., Feng, L., Zhang, M. H., Qin, D., Jiang, J., Cheng, X. D., et al. (2014). New metabolite profiles of Danshensu in rats by ultraperformance liquid chromatography/quadrupole-time-of-flight mass spectrometry. J. Chromatogr. $B$ Analyt. Technol. Biomed. Life Sci. 955-956, 20-25. doi: 10.1016/j.jchromb.2014.02.010

Guo, J., Zhang, Y., Zeng, L., Liu, J., Liang, J., and Guo, G. (2013). Salvianic acid a protects L-02 cells against gamma-irradiation-induced apoptosis via the scavenging of reactive oxygen species. Environ. Toxicol. Pharmacol. 35, 117-130. doi: 10.1016/j.etap.2012.11.010

Jia, D., Li, T., Chen, X., and Ding, X. (2018). Salvianic acid a sodium protects HUVEC cells against tert-butyl hydroperoxide induced oxidative injury via mitochondria-dependent pathway. Chem. Biol. Interact. 279, 234-242. doi: 10.1016/j.cbi.2017.10.025

Jiang, J. J., Zhao, X., Li, X. X., Wu, S. Y., Yu, S. D., Lou, Y. F., et al. (2017). High-throughput determination of sodium danshensu in beagle dogs by the LCMS/MS method, employing liquid-liquid extraction based on 96-well format plates. Molecules 22, 667-681. doi: 10.3390/molecules22050667

Kang, D. G., Yun, Y. G., Ryoo, J. H., and Lee, H. S. (2002). Anti-hypertensive effect of water extract of danshen on renovascular hypertension through inhibition of the renin angiotensin system. Am. J. Chin. Med. 30, 87-93. doi: 10.1142/S0192415X02000107

Li, W., Li, Z. W., Han, J. P., Li, X. X., Gao, J., and Liu, C. X. (2008). Determination and pharmacokinetics of danshensu in rat plasma after oral administration of danshen extract using liquid chromatography/tandem mass spectrometry. Eur. J. Drug Metab. Pharmacokinet. 33, 9-16. doi: 10.1007/BF03191013

Li, Y. G., Song, L., Liu, M., Hu, Z. B., and Wang, Z. T. (2009). Advancement in analysis of Salviae miltiorrhizae radix et Rhizoma (Danshen). J. Chromatogr. A 1216, 1941-1953. doi: 10.1016/j.chroma.2008.12.032

Liu, Y. Q., Cai, Q., Liu, C., Bao, F. W., and Zhang, Z. Q. (2014). Simultaneous determination and pharmacokinetic comparisons of multi-ingredients after
Laboratory Animal Science (ICLAS) and the Councils for International Organizations of Medical Sciences (CIOMS) in 2012.

\section{AUTHOR CONTRIBUTIONS}

$\mathrm{XM}$ and JJ preformed the bioanalysis and prepared the manuscript. HP established the analytical method and revised the manuscript. SWa performed the animal experiment. SWa analyzed data and calculated PK parameters. GF and YL designed the whole research and interpreted results of experiments. GF reviewed the final manuscript, and all the authors have read and approved the final version.

\section{FUNDING}

This work was supported by the Project Plan of Important Developing Discipline Construction, China (No. 2016ZB0302) and Funding scheme of young teachers in Shanghai universities (ZZJKYX16009).

oraladministration of radix salviae miltiorrhizae extract, hawthorn extract, and a combination of both extracts to rats. J. Anal. Methods Chem. 2014:617367. doi: 10.1155/2014/617367

Liu, Y., Li, X., Li, Y., Wang, L., and Xue, M. (2010). Simultaneous determination of danshensu, rosmarinic acid, cryptotanshinone, tanshinone IIa, tanshinone I and dihydrotanshinone I by liquid chromatographic-mass spectrometry and the application to pharmacokinetics in rats. J. Pharm. Biomed. Anal. 53, 698-704. doi: 10.1016/j.jpba.2010.03.041

Shen, Y., Wang, X., Xu, L., Liu, X., and Chao, R. (2009). Characterization of metabolites in rat plasma after intravenous administration of salvianolic acid a by liquid chromatography/time-of-flight mass spectrometry and liquid chromatography/ion trap mass spectrometry. Rapid Commun. Mass Spectrom. 23, 1810-1816. doi: 10.1002/rcm.4078

Song, Q., Chu, X., Zhang, X., Bao, Y., Zhang, Y., Guo, H., et al. (2016). Mechanisms underlying the cardioprotective effect of Salvianic acid a against isoproterenol-induced myocardial ischemia injury in rats: possible involvement of L-type calcium channels and myocardial contractility. J. Ethnopharmacol. 189, 157-164. doi: 10.1016/j.jep.2016.05.038

Su, C. Y., Ming, Q. L., Rahman, K., Han, T., and Qin, L. P. (2015). Salvia miltiorrhiza: traditional medicinal uses, chemistry, and pharmacology. Chin. J. Nat. Med. 13, 163-182. doi: 10.1016/S1875-5364(15)30002-9

Wang, X., Li, W., Ma, X., Yan, K., Chu, Y., Han, M., et al. (2015). Identification of a major metabolite of danshensu in rat urine and simultaneous determination of danshensu and its metabolite in plasma: application to a pharmacokinetic study in rats. Drug Test. Anal. 7, 727-736. doi: 10.1002/ dta. 1750

Wang, L., Ma, R., Liu, C., Liu, H., Zhu, R., Guo, S., et al. (2017). Salvia miltiorrhiza: a potential red light to the development of cardiovascular diseases. Curr. Pharm. Des. 23, 1077-1097. doi: 10.2174/1381612822666161010105242

Wang, C. Y., Ma, F. L., Liu, J. T., Tian, J. W., and Fu, F. H. (2007). Protective effect of salvianic acid a on acute liver injury induced by carbon tetrachloride in rats. Biol. Pharm. Bull. 30, 44-47. doi: 10.1248/bpb.30.44

Wang, L., and Sweet, D. H. (2013). Competitive inhibition of human organic anion transporters 1 (SLC22A6), 3 (SLC22A8) and 4 (SLC22A11) by major components of the medicinal herb Salvia miltiorrhiza (Danshen). Drug Metab. Pharmacokinet. 28, 220-228. doi: 10.2133/dmpk.DMPK-12-RG-116

Wang, X. J., and Xu, J. X. (2005). Salvianic acid a protects human neuroblastoma SH-SY5Y cells against MPP+-induced cytotoxicity. Neurosci. Res. 51, 129-138. doi: 10.1016/j.neures.2004.10.001

Wei, Z. Z., Chen, D., Liu, L. P., Gu, X., Zhong, W., Zhang, Y. B., et al. (2018). Enhanced neurogenesis and collaterogenesis by sodium danshensu treatment 
after focal cerebral ischemia in mice. Cell Transplant. 27, 622-636. doi: $10.1177 / 0963689718771889$

Xu, H., Li, Y., Che, X., Tian, H., Fan, H., and Liu, K. (2014). Metabolism of salvianolic acid a and antioxidant activities of its methylated metabolites. Drug Metab. Dispos. 42, 274-281. doi: 10.1124/dmd.113.053694

Yu, P. F., Wang, W. Y., Eerdun, G., Wang, T., Zhang, L. M., Li, C., et al. (2011). The role of P-glycoprotein in transport of Danshensu across the blood-brain barrier. Evid. Based Complement. Alternat. Med. 8, 1-5. doi: $10.1155 / 2011 / 713523$

Zhan, Y., Xu, J. P., Liang, J. B., Sheng, L. S., Xiang, B. R., Zou, Q. G., et al. (2008). Simultaneous LC-MS-MS analysis of danshensu, salvianolic acid B, and hydroxysafflor yellow a in beagle dog plasma, and application of the method to a pharmacokinetic study of Danhong lyophilized powder for injection. Chromatographia 68, 71-79. doi: 10.1365/s10337-008-0652-0

Zhang, N., Zou, H., Jin, L., Wang, J., Zhong, M. F., Huang, P., et al. (2010). Biphasic effects of sodium danshensu on vessel function in isolated rat aorta. Acta Pharmacol. Sin. 31, 421-428. doi: 10.1038/aps.2010.24

Zhao, Q. T., Guo, Q. M., Wang, P., and Wang, Q. (2012). Salvianic acid A inhibits lipopolysaccharide-induced apoptosis through regulating glutathione peroxidase activity and malondialdehyde level in vascular endothelial cells. Chin. J. Nat. Med. 10, 53-57. doi: 10.1016/S1875-5364(12)60012-0

Zhao, D., Liu, H., Wang, F., Feng, Q., and Li, M. (2013). A simple, but highly sensitive, graphene-based voltammetric sensor for salvianic acid a sodium. Anal. Sci. 29, 625-630. doi: 10.2116/analsci.29.625
Zheng, L., Gong, Z., Lu, Y., Xie, Y., Huang, Y., Liu, Y., et al. (2015). A UPLCMS/MS method for simultaneous determination of danshensu, protocatechuic aldehyde, rosmarinic acid, and ligustrazine in rat plasma, and its application to pharmacokinetic studies of Shenxiong glucose injection in rats. J. Chromatogr. B Analyt. Technol. Biomed. Life Sci. 997, 210-217. doi: 10.1016/j. jchromb.2015.06.008

Zhou, L., Chow, M. S., and Zuo, Z. (2009). Effect of sodium caprate on the oral absorptions of danshensu and salvianolic acid B. Int. J. Pharm. 379, 109-118. doi: 10.1016/j.ijpharm.2009.06.016

Conflict of Interest Statement: The handling editor declared a shared affiliation, though no other collaboration, with the authors, GF and SWa.

The remaining authors declare that the research was conducted in the absence of any commercial or financial relationships that could be construed as a potential conflict of interest.

Copyright (C) 2019 Meng, Jiang, Pan, Wu, Wang, Lou and Fan. This is an openaccess article distributed under the terms of the Creative Commons Attribution License (CC BY). The use, distribution or reproduction in other forums is permitted, provided the original author(s) and the copyright owner(s) are credited and that the original publication in this journal is cited, in accordance with accepted academic practice. No use, distribution or reproduction is permitted which does not comply with these terms. 